\title{
Felt Sandwich Exclusion for Apical Multiple Muscular Ventricular Septal
}

\author{
Kota Agematsu ${ }^{1}$, Masahiro Kaneko ${ }^{2}$, and Yoshiharu Nishimura ${ }^{3}$ \\ ${ }^{1}$ Wakayama Medical University \\ ${ }^{2}$ Affiliation not available \\ ${ }^{3}$ Wakayama Kenritsu Ika Daigaku
}

June 9, 2021

\begin{abstract}
Apical multiple muscular ventricular septal defects (VSDs) are difficult to visualize, and closing these VSDs is technically troublesome. For these reasons, the surgical outcome of the lesion is unsatisfactory. Some surgical techniques have been reported to improve the outcome, but they are not definite. We present a case with atrial septal defect, multiple muscular and perimembranous VSDs, and pulmonary hypertension due to high pulmonary blood flow. In the first operation during the infantile period, the patient received closure of the perimembranous and muscular VSD through the right atrium and atrial septal defect closure. However, postoperative pulmonary blood flow was not decreased compared with that of before the operation, and pulmonary hypertension was also not improved. In addition to these lesions, tricuspid valve insufficiency was also observed after the operation. Postoperative computed tomography showed channels of apical muscular VSDs in the apex of the right ventricle. In the second operation, exclusion of apex muscular VSDs using the felt sandwich technique and tricuspid valve repair were performed. After closing multiple muscular VSDs during the operation, pulmonary blood flow was significantly decreased, and pulmonary hypertension was improved as well. After the second operation, postoperative echocardiography and catheter examination showed decreased VSD shunt and pulmonary blood flow, resulting in improved pulmonary hypertension.
\end{abstract}

Title page

Felt Sandwich Exclusion for Apical Multiple Muscular Ventricular Septal Defects

Kota Agematsu, Mitsugi Nagashima, Masahiro Kaneko,

Yoshiharu Nishimura

Department of Cardiovascular Surgery, Wakayama Medical University, 811-1, kimiidera, wakayama-city, wakayama, Japan

Corresponding author Kota Agematsu

No funding is related to this case report

No disclosure of conflict of interest

The Institutional Review Board in our institute has reviewed this case report and approved publication

Informed consent has been obtained from the patient's family

Introduction

Surgical treatment for apical muscular ventricular septal defects (VSDs) remains challenging, because there are many variations in the location where the VSDs open, and it is hard to visualize and close VSDs using 
the transatrial approach, especially in small body patients. For some patients with muscular VSDs that cannot be closed, pulmonary artery banding is indicated, but the outcome of this procedure is uncertain. Some surgical closure techniques for VSDs have been reported, but the outcomes of these techniques for the lesion are also not definitive [1-3]. In this report, we present the clinical case of a patient who received effective surgical treatment for apical muscular VSDs.

Clinical summary

A 5-month-old girl who weighed $4.98 \mathrm{~kg}$ had been diagnosed with perimembranous and muscular VSDs, atrial septal defect (ASD), and pulmonary hypertension due to high pulmonary blood flow. Preoperative catheter examination showed that the ratio of pulmonary to systemic blood flow (Qp/Qs) was 2.3 and pulmonary to systemic blood pressure ratio was 0.86 . This patient received transatrial VSDs closure, both perimembranous and muscular, as well as ASD closure in the first operation. Postoperative catheter examination revealed a Qp/Qs of 2.4 and sustained pulmonary hypertension. Postoperative echocardiography indicated moderate tricuspid valve regurgitation and residual ASD. To relieve pulmonary hypertension, we performed a second operation almost 2 months after the first operation. We planned to carry out muscular VSDs exclusion closure, tricuspid valve repair, and ASD closure. In case of uncontrolled pulmonary blood flow by VSD exclusion, we also planned for additional pulmonary artery banding. Before surgery, we roughly detected the channels of the VSD in the cardiac apex using computed tomography (Figure 1) and estimated part of the exclusion area. During the second operation, we established cardiopulmonary bypass and obtained cardiac arrest. We repaired the tricuspid valve and also directly closed the residual ASD. We did not visualize the muscular VSDs through the right atrium, and closing the VSDs through the right atrium was technically challenging. To exclude the muscular VSDs, we performed the felt sandwich technique on the cardiac apex where channels of VSDs were located (Figure 2). After aortic de-clamping, normal heart rhythm was obtained, and weaning from cardiopulmonary bypass was uneventful. Transesophageal echocardiography showed a reduced trans-VSD shunt as compared with that presented before the closure of VSDs. After the patient was weaned from cardiopulmonary bypass, her Qp/Qs was less than 1.2, and pulmonary band was not performed. The patient was discharged from the hospital without any complications. Postoperative echocardiography showed a tiny muscular VSD shunt and mild pulmonary hypertension, and postoperative catheter examination showed a Qp/Qs of 1.02 and mild pulmonary hypertension, in which the ratio of pulmonary arterial pressure to systemic pressure was 0.4 . the patient was placed on antipulmonary hypertensive medication.

\section{Discussion}

The optical diagnosis and treatment of muscular VSDs remains indeterminate. Muscular VSDs exist on various parts of the ventricular septum, and it is hard to visualize the VSD channel in the coarse trabeculations of the right ventricle, not only on preoperative images but also via direct vision during the operation. However, computed tomography images obtained before the operation are useful as a reference, and in our case, a surgical strategy of apical VSDs sandwich exclusion could be planned while referring to the preoperative image.

Although some surgical approaches, including intraoperative device closure [4] and the sandwich technique, have been reported, optimal outcomes have not yet been obtained. Device-related complications such as device embolization, tricuspid or aortic valve damage, and recurrent defects may occur. The ventricle septal sandwich technique is simple and effective; however, the use of numerous felt patches on the ventricular septum disturbs the movement of the ventricular septum, resulting in impairing ventricular function [2]. In addition to surgical techniques, visualization of muscular VSDs is an important factor in VSD treatment. Apical right or left ventriculotomy has been used to visualize the apical muscular VSDs for closure [5], but ventriculotomy might lead to late ventricular dysfunction, aneurysmal formation, and ventricular arrhythmias in the future.

Because muscular VSDs can be located anywhere on the ventricular septum, a definitive surgical closure method cannot be established. The choice of surgical technique should be based on where the VSDs are 
located. In determining the best surgical technique, it is important to determine preoperatively where the VSD channels are. In our presented case, computed tomography was useful for locating the VSDs and which channels were near the ventricular apex, and these VSDs were estimated to be excluded by the using felt sandwich technique exteriorly. This technique may be one choice for surgical closure for muscular VSDs.

\section{References}

1.Kitagawa T, Durham III LA, Mosca RS, Bove EL Techniques and results in the anagement of multiple ventricular septal defects. J Thorac Cardiovasc Surg 1997;115:848-56

2.Ootaki Y, Yamaguchi M, Yoshimura N, Oka S, Yoshida M, Hasegawa T Surgical management of trabecular ventricular septal defects: The sandwich technique. J Thorac Cardiovasc Surg 2003;125:508-12

3.Yoshimura N, Matsuhisa H, Otaka S, Takuro MIsaki, et.al Surgical management of multiple ventricular septal defect:The role of the felt sandwich technique. J Thorac Cardiovasc Surg 2009;137:924-8

4. Okubo M, Benson LN, Nykanen D, Azakie A, Van Arsdell G, Coles J, et al. Outcomes of intraoperative device closure of muscular ventricular septal defects. Ann Thorac Surg. 2001;72:416-23.

5. Myhre U, Duncan BW, Mee RB, Joshi R, et al Apical right ventriculotomy for closure of apical ventricular septal defects. Ann Thorac Surg 2004;78:204-8.
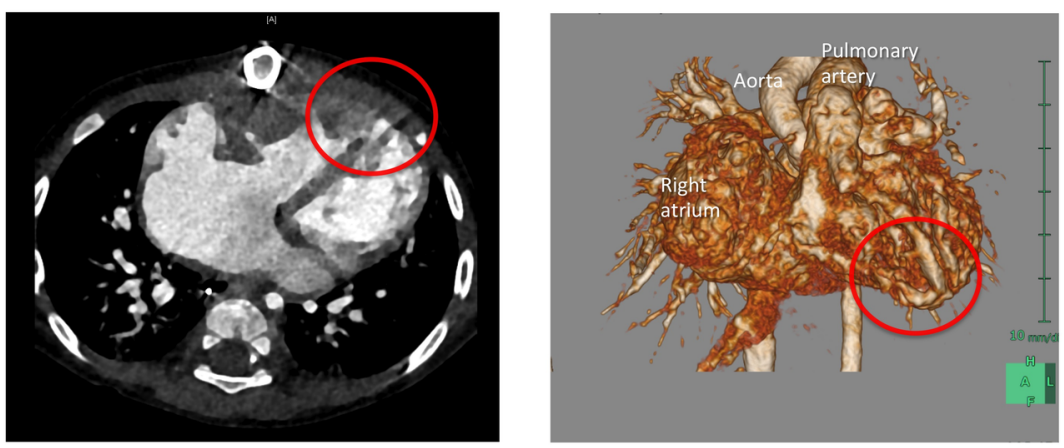

Figure 1 


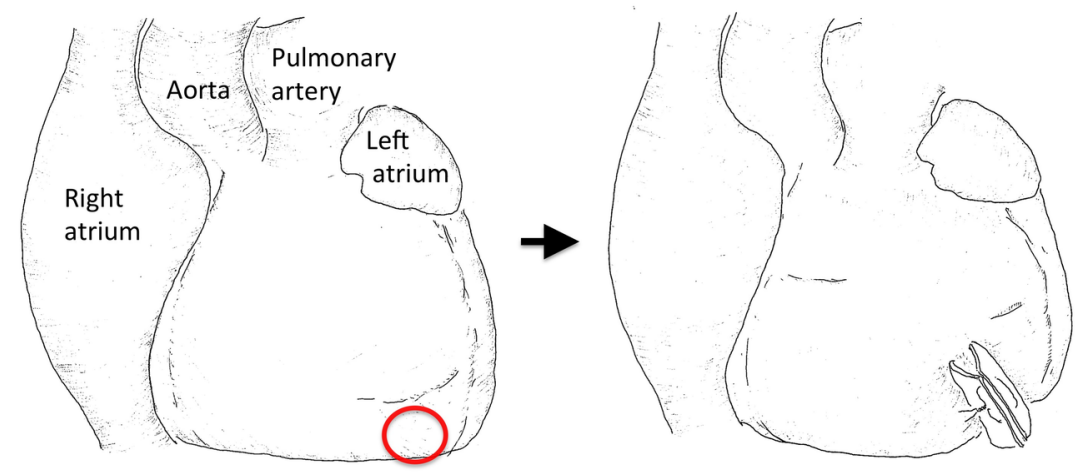

Figure 2 